\title{
Correction to: Sca-1-Positive Cardiac Stem Cell Migration in a Cardiac Infarction Model
}

\author{
Jingjin Liu,, ${ }^{1,2}$ Yongshun Wang, ${ }^{1,2}$ Wenjuan Du, ${ }^{1,2}$ and Bo Yu ${ }^{1,2,3}$
}

Correction to: Inflammation (2013) 36(3):738-49

https://doi.org/10.1007/s10753-013-9600-8

After publication of our article [1], we became aware that there were errors in Fig. 1, namely that the Echocardiographic assessment of $1 \mathrm{w}, 2 \mathrm{w}$, and $4 \mathrm{w}$ groups was incorrectly presented. These errors do not affect the discussion or conclusions in the article. The correct versions of Fig. 1 are shown below. We apologize to the journal and to the readers for this error.

Jingjin Liu and Yongshun Wang contributed equally to this work.

The online version of the original article can be found at https://doi.org/ $10.1007 / \mathrm{s} 10753-013-9600-8$

\footnotetext{
${ }^{1}$ Key Laboratories of Education Ministry for Myocardial Ischemia Mechanism and Treatment, Harbin, China

${ }^{2}$ Depatment of Cardiology, Second Affiliated Hospital of Harbin Medical University, Harbin, 150086, China

${ }^{3}$ To whom correspondence should be addressed at Depatment of Cardiology, Second Affiliated Hospital of Harbin Medical University, Harbin, 150086, China. E-mail: yubodr@163.com
}

\section{REFERENCE}

1. Jingjin Liu, Yongshun Wang, Wenjuan Du, Bo Yu. 2013. Sca-1positive cardiac stem cell migration in a cardiac infarction model. Inflammation 36(3):738-749. https://doi.org/10.1007/s10753-0139600-8. 
Baseline
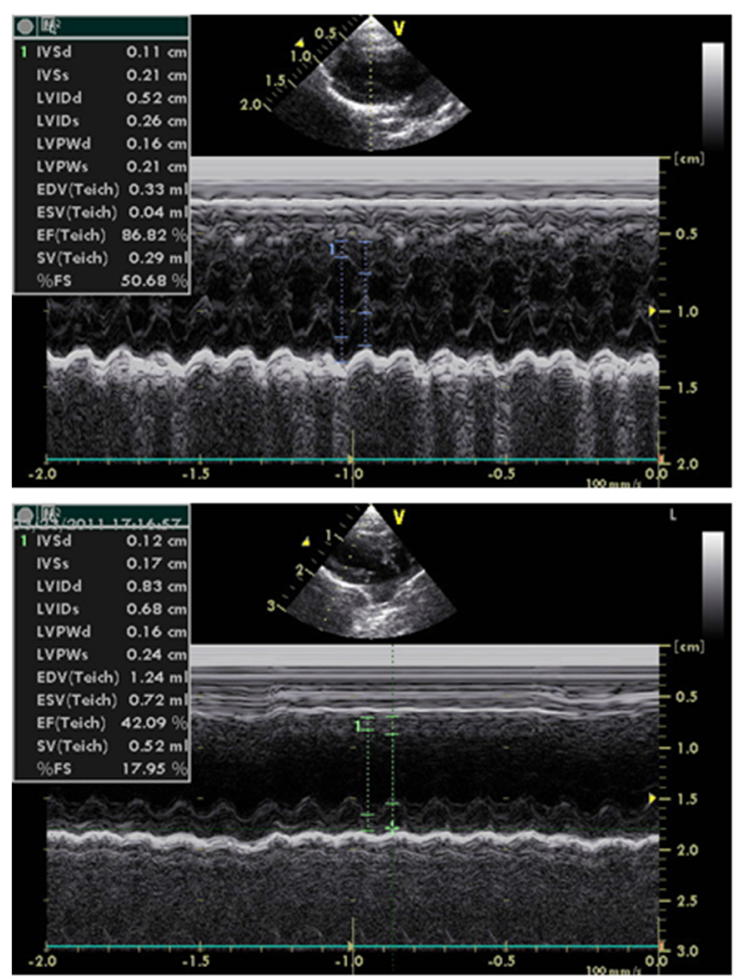

$2 \mathbf{W}$

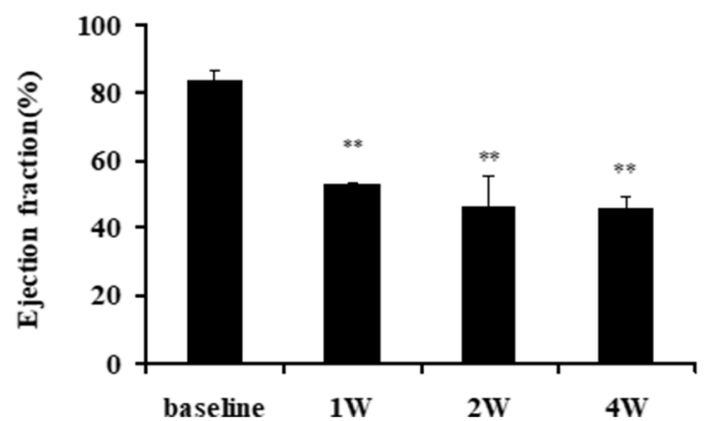

$1 \mathrm{~W}$
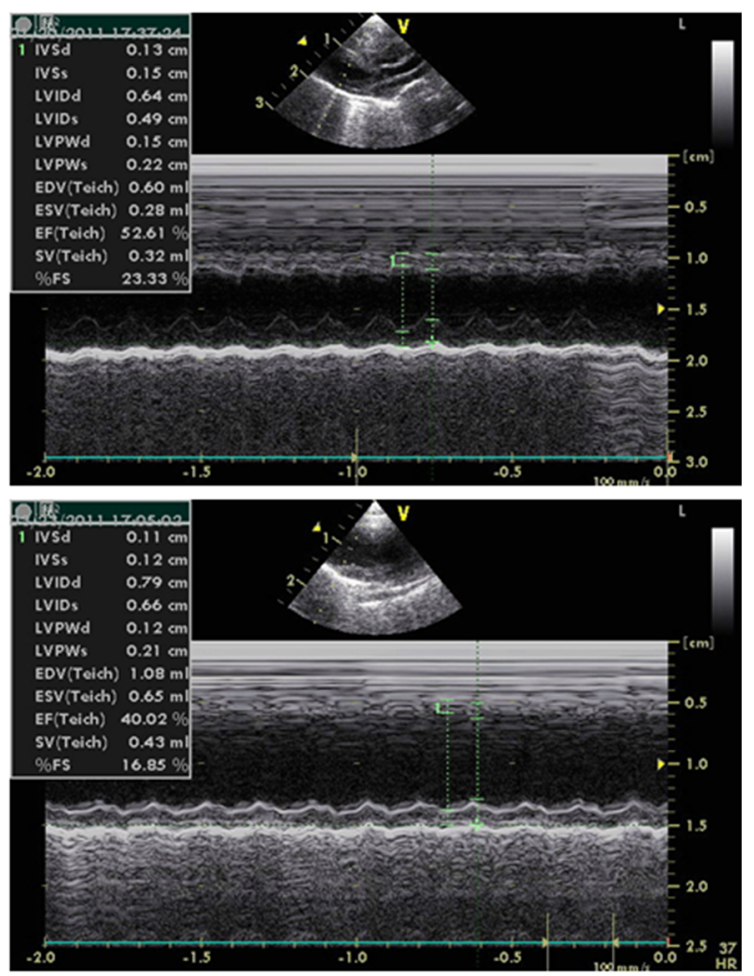

$4 \mathrm{~W}$

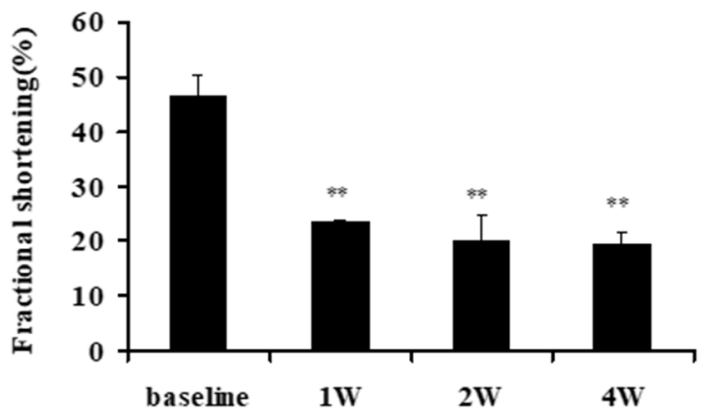

Fig. 1. Echocardiographic assessment of cardiac function. Representative M-mode echocardiograms are shown at baseline (normal rats) and in the $1 \mathrm{w}, 2 \mathrm{w}$, and $4 \mathrm{w}$ groups. In the groups that had surgery, 1,2 , and 4 weeks after ligation of the $\mathrm{LAD}$, the left ventricular ejection fraction (LVEF) declined to $52.88 \pm$ $0.71 \%, 46.51 \pm 8.58 \%$, and $45.49 \pm 4.05 \%$, respectively, compared with the baseline $(83.45 \pm 2.99 \%, p<0.01)$. The left ventricular fractional shortening (LVFS) declined to $23.47 \pm 0.48 \%, 20.20 \pm 4.54 \%$, and $19.49 \pm 1.94 \%, 1,2$, and 4 weeks after AMI, respectively, compared with the baseline (46.66 \pm $3.57 \%, p<0.01)$. $* * p \leq 0.001$, compared to the baseline group; $n=8$ for each group. 